\title{
Dinâmica da vegetação campestre sob diferentes tratamentos de adubação e manejo do pastoreio na Região do Alto Camaquã, RS.
}

\author{
Dynamic of grassland under different treatments of fertilization and grazing \\ management at Alto Camaqua, RS.
}

\author{
Lidiane da Rosa Boavista*1, Fernando Luiz Ferreira de Quadros ${ }^{2}$, \\ José Pedro Pereira Trindade ${ }^{3,}$ Marcos Flávio Silva Borba ${ }^{4}$ \\ ${ }^{1}$ Doutoranda em Ecologia, Universidade Federal do Rio Grande do Sul, Porto Alegre, Brasil \\ ${ }^{2}$ Doutor em Zootecnia, Professor associado do Departamento de Zootecnia, Universidade Federal de Santa Maria, \\ Santa Maria, Brasil \\ ${ }^{3}$ Doutor em Zootecnia \\ ${ }^{4}$ Doutor em Sociologia e Agroecologia Brasil
}

\begin{abstract}
Resumo
Foi avaliada a dinâmica vegetacional de uma pastagem natural submetida aos fatores de manejo adubação e pastejo, em quatro locais diferentes, nos municípios de Pinheiro Machado e Piratini, localizados no terço superior da bacia do Rio Camaquã. Os tratamentos foram constituídos pela associação desses fatores. A estimativa da composição de espécies e suas respectivas biomassas seguiram os procedimentos de campo do método BOTANAL, com algumas adaptações, juntamente com a composição de espécies foram descritos os tipos funcionais de vegetação presentes, utilizando atributos foliares, como área foliar específica e teor de matéria seca. Foi observada uma mudança na dinâmica vegetacional em função da interação entre períodos de avaliação, adubação e pastoreio, mostrando uma divergência das trajetórias da vegetação. Não foi evidenciada uma associação nítida entre os tipos funcionais e as variáveis ambientais.
\end{abstract}

Palavras-chave: pastagem natural, biomassa, tipos funcionais, espécies.

\begin{abstract}
We evaluated the dynamics vegetation of grassland subjected to management factors fertilization and grazing, at four different locations in the counties of Pinheiro Machado and Piratini, located in the upper basin of the River Camaqua. The treatments were a combination of these factors. The estimate of the species composition and their biomass followed the procedures of the method BOTANAL field, with some adaptations, together with the composition of species have been described functional types of vegetation present, using leaf traits such as leaf area matter content and specific dry. There was a change in vegetation dynamics due to the interaction between evaluation periods, fertilization and grazing, showing a divergence of trajectories of vegetation. There was found a clear association between the functional types and environmental variables.
\end{abstract}

Keywords: grassland, biomass, functional types, species 


\section{Introdução}

$\mathrm{A}$ s pastagens naturais constituem um dos maiores biomas do mundo, representando a principal fonte de alimento para os rebanhos, principalmente bovinos e ovinos, da região (Carvalho et al., 2006), e podem ser caracterizadas por uma cobertura vegetal com predomínio de gramíneas, com pouca ou sem presença de árvores. A diversidade de espécies neste ecossistema campestre é considerável e única, existindo cerca de 2.200 espécies campestres, sendo 450 gramíneas, 450 compostas e 200 leguminosas (Boldrini, 2009).

A composição florística das pastagens naturais é composta principalmente por espécies estivais que diminuem seu crescimento no período hibernal, ocasionando uma diminuição na sua produtividade em épocas frias do ano (Pellegrini et al., 2010). Em vista disto, a pastagem natural requer certos manejos para solucionar este problema, a adubação é um usual elemento para promover uma melhora na produção de forragem (Carvalho et al., 2006), o que juntamente com outras práticas benéficas de manejo proporciona muitos ganhos para os produtores.

Considerando toda diversidade, característica desse ecossistema, e a dificuldade de identificação das espécies, estas podem ser classificadas em tipos funcionais (TFs), que são grupos de plantas que exibem respostas similares às condições de ambiente e efeitos parecidos sobre processos do ecossistema. $\mathrm{O}$ agrupamento dos TFs, ou seja, o agrupamento de plantas funcionalmente similares, pode permitir uma percepção mais nítida da associação entre vegetação e ambiente (Lavorel e Garnier, 2002).

A correção de acidez e a elevação do nível de fertilidade são alternativas para aumentar o rendimento e a qualidade de forragem, sendo também importantes fatores que influenciam modificações da composição florística das pastagens (Pinto et al., 2013), O fato dos ecossistemas terem variabilidade na disponibilização de nutrientes, bem como na distribuição e intensidade do pastejo, faz com que existam sítios com diferentes combinações de fertilidade e pressão de pastejo o que heterogeneidade espacial da vegetação (Boldrini, 2009).

Um ponto importante para o sucesso das pastagens naturais é o conhecimento das principais espécies componentes da vegetação, suas respostas frente ao ambiente e ao manejo (Boldrini, 2009).

O conhecimento da dinâmica vegetacional campestre é muito relevante, quando se busca melhoria em estratégias de manejo, visando uma melhor utilização e conservação da diversidade, com isso o objetivo deste trabalho foi avaliar a dinâmica da vegetação em resposta aos fatores adubação e pastejo, através da composição botânica e tipos funcionais, em quatro áreas de vegetação campestre localizadas na região do Alto Camaquã.

\section{Material e métodos}

O trabalho foi realizado em quatro áreas localizadas na porção superior da bacia do Rio Camaquã, nos municípios de Pinheiro Machado e Piratini, RS, no período de 2008 a 2011.

A região fisiografica é a Serra do Sudeste, embasada pelo substrato granítico do Escudo Cristalino Sul-RioGrandense. O clima da região, segundo a classificação de Köppen, é o temperado úmido ( $\mathrm{Cfb}$ ), com temperatura do ar média anual de $16^{\circ} \mathrm{C}$ e precipitação média anual em torno de $1380 \mathrm{~mm}$.

São áreas de campo natural, de pecuaristas familiares, que fazem parte do projeto Alto Camaquã da Embrapa CPPSUL, Bagé, RS e estão organizadas em Unidades Experimentais Participativas (UEPAS).

As áreas foram subdivididas, com cercas eletrificadas e os níveis de adubação foram 2 t/ha de pó de rocha (Pó de Rocha Bioland ${ }^{\circledR}$ ) e $500 \mathrm{Kg} / \mathrm{ha}$ de Fosfato Natural Reativo - Bayóvar (Sechura) e calcário. O arranjo experimental foi em blocos (UEPAS), sendo as unidades experimentais os transectos e amostrais (quadros dentro de cada transecto).

Caracterização das áreas: Áreas 1 (O) e 4 (R), com 10 ha $^{-1}$ cada uma, quanto a fertilização foram adubadas com fosfato natural e calcário e para os pastejos foram contínuos e rotativos, estes dois fatores que compuseram os tratamentos e foram aleatorizados nas 14 repetições.

Áreas 2 (S) e $3(\mathrm{C}), 5$ ha $^{-1}$ e 4 ha $^{-1}$ respectivamente, quanto a fertilização foram adubadas com fosfato natural e pó-de-rocha e para os pastejos foram contínuos e rotativos, estes dois fatores que compuseram os tratamentos e foram aleatorizados nas 14 repetições. Para os levantamentos da vegetação dentro dos tratamentos, foram alocados transectos de $2 \mathrm{~m}$ de comprimento por $0.50 \mathrm{~m}$ de largura, totalizando 14 transectos em cada área de estudo, totalizando 56 transectos no total das quatro áreas, sendo que os tratamentos foram aplicados aos pares, onde áreas 1 e 4 receberam um tipo de tratamento e áreas 2 e 3 outro tipo de tratamento, cada área representa um bloco, sendo esta, o fator de bloqueamento nas posteriores análises estatísticas.

Foi descrita a composição florística através da listagem das espécies componentes e respectivas quantidades de biomassa por estimativa visual. As amostragens foram adaptadas ao método Botanal (Tothill et al., 1992). Os dados foram anotados em planilha de campo e posteriormente repassados a planilha eletrônica de cálculos automatizada (Martins e Quadros, 2004), com adaptações de (Martins et al., 2007). Visando gerar hipóteses sobre os efeitos dos tratamentos, foram utilizadas análises de ordenação através de coordenadas principais (PCOA), sendo a medida de semelhança a distância de corda e para a análise de agrupamento dos tratamentos utilizou-se o método da soma de quadrados (Ward) a partir da 
distância de corda. Para analisar e testar a significância das diferenças entre os tratamentos para a composição e dinâmica de espécies, foram feitas análises de aleatorização, todas as análises foram feitas com o auxilio do software MULTIV (Pillar, 2009).

Concomitantemente às amostragens de identificação das espécies, foram coletadas lâminas foliares das gramíneas que tiverem a sua participação superior a $3 \%$ na massa total de forragem. Nessas lâminas, foram determinados os atributos de teor de matéria seca (TMS) e área foliar específica (AFE), seguindo a metodologia proposta por Garnier et al. (2001), Cornelissen et al. (2003) e Ansquer, et al. (2004). Nessa metodologia, os afilhos coletados serão acondicionados em recipientes plásticos, com o cuidado de não danificar a lâmina foliar, devidamente identificados, contendo água deionizada até seu terço inferior. Após, foram acondicionados em caixa de isopor com gelo para serem transportados até o refrigerador. As amostras deveriam permanecer no refrigerador por um período mínimo de seis horas ou até $24 \mathrm{~h}$, sob temperatura de $2-6^{\circ} \mathrm{C}$ com escuridão total para a padronização das amostras até o momento de separação. Após este período, as folhas adultas íntegras (saudáveis e não pastejadas) dos afilhos coletados, foram separadas, pesadas para a obtenção do peso verde e digitalizadas com scanner de mesa para a determinação da área foliar. Posteriormente, as folhas foram acondicionadas em embalagens de papel e levadas à estufa de ar forçado a $65^{\circ} \mathrm{C}$, por um período de 72 horas. Após a secagem, as amostras foram pesadas, obtendo-se o peso da massa seca da amostra. O TMS foi obtido pela razão entre o peso seco e o peso verde das folhas sendo valor final expresso em g. $\mathrm{Kg}^{-1}$ e a AFE foi determinada pela razão da área foliar com o peso seco (expresso em m $\mathrm{Kg}^{-1}$ ), sendo esta obtida com auxílio do sofware Image J (Rasband, 2007).

\section{Resultados e discussão}

As análises de ordenação e agrupamento dos tratamentos através dos levantamentos realizados logo após a aplicação dos tratamentos e dois anos após o acompanhamento inicial da composição botânica originaram os diagramas de ordenação baseados na massa de forragem (MF) por espécie, esses valores (em kg MS ha-1) de todas as espécies componentes e o descritor material morto foram considerados na análise multivariada dos dados e são os que geram a dispersão dos tratamentos nos planos de ordenação (Figuras 1 e 2).

No estudo da dinâmica vegetacional ao longo dos quatro anos de avaliação procurou-se detectar padrões de variação. Nas duas avaliações realizadas na área experimental, foram registradas 113 espécies pertencentes a 19 famílias, descritas em transectos fixos, para observação da dinâmica temporal e espacial das áreas em diferentes épocas.

Análise de variância através de testes de aleatorização, comparando os tratamentos quando a contribuição de espécies indicou interação significativa entre manejo/adubação $(\mathrm{P}=0,002)$ e época/adubação $(\mathrm{P}=0,001)$. Podemos observar que houve diferença na dinâmica da vegetação através da avaliação das biomassas das espécies, em função dos tratamentos, mesmo pelo pouco tempo de introdução do experimento, como também demonstra Ferreira et al. 2011 e Liancourt, 2005, considerando que alguns autores afirmam que a composição só se modifica depois de alguns anos de estudo (Liancourt, 2005).

Na Figura 1, estão representadas as trajetórias da dinâmica vegetacional, em função da época e adubação, o eixo 1 sintetizou $49 \%$ e o eixo 2 sintetizou $33 \%$, o que representa $82 \%$ da variação total. Pode ser observado que no levantamento inicial, existe uma condição da vegetação muito similar em todos os tratamentos, tendo todas as trajetórias seu início no mesmo quadrante, que posteriormente divergem para trajetórias bem nítidas, sendo que dos quatro tratamentos dois tendem a direções diversas em função da composição botânica. Isto indica que houve uma tendência da comunidade vegetal dos tratamentos a apresentar uma composição florística diferenciada.

A condição de similaridade inicial se deve provavelmente ao histórico de manejo das áreas, que sempre foram manejadas em sistema de pastoreio contínuo e sem controle, onde o manejo da vegetação sempre foi o mesmo para todas as estações do ano e o diferimento nunca havia sido aplicado.

No início dos levantamentos, pode ser observada a maior contribuição de espécies bem adaptadas a pastejos constantes e caracterizados por uma seletividade dos animais, de forma não controlada, contrariamente ao que ocorreu quando as áreas foram subdividas, onde houve um maior controle sobre o consumo de espécies dentro de cada potreiro, resultados semelhantes aos descritos por Schönback et al., 2010.

Esta condição inicial foi representada por uma maior contribuição das espécies Erygium horridum, Oxalis articulata, Hypoxis decumbens, Dichondra sericea e Relbunium richardianum, este último mostrou-se altamente associado à espécie E. horridum (caraguatá), e a espécie Aristida uruguayensis. Isso se explica pelo fato do caraguatá formar grandes touceiras de folhas rosetadas e espinescentes, que consequentemente servem de proteção para espécies, dificultando o acesso dos animais (Flores e Jurado, 2003), o mesmo ocorrendo com $A$. uruguayensis, mesmo não sendo registradas touceiras tão densas, essas também apresentam função de proteção.

Na segunda época de avaliação, segundo as trajetórias o tratamento com calcário demonstrou-se associado ao aumento do componente material morto. Isto se deve por uma maior contribuição da espé- 


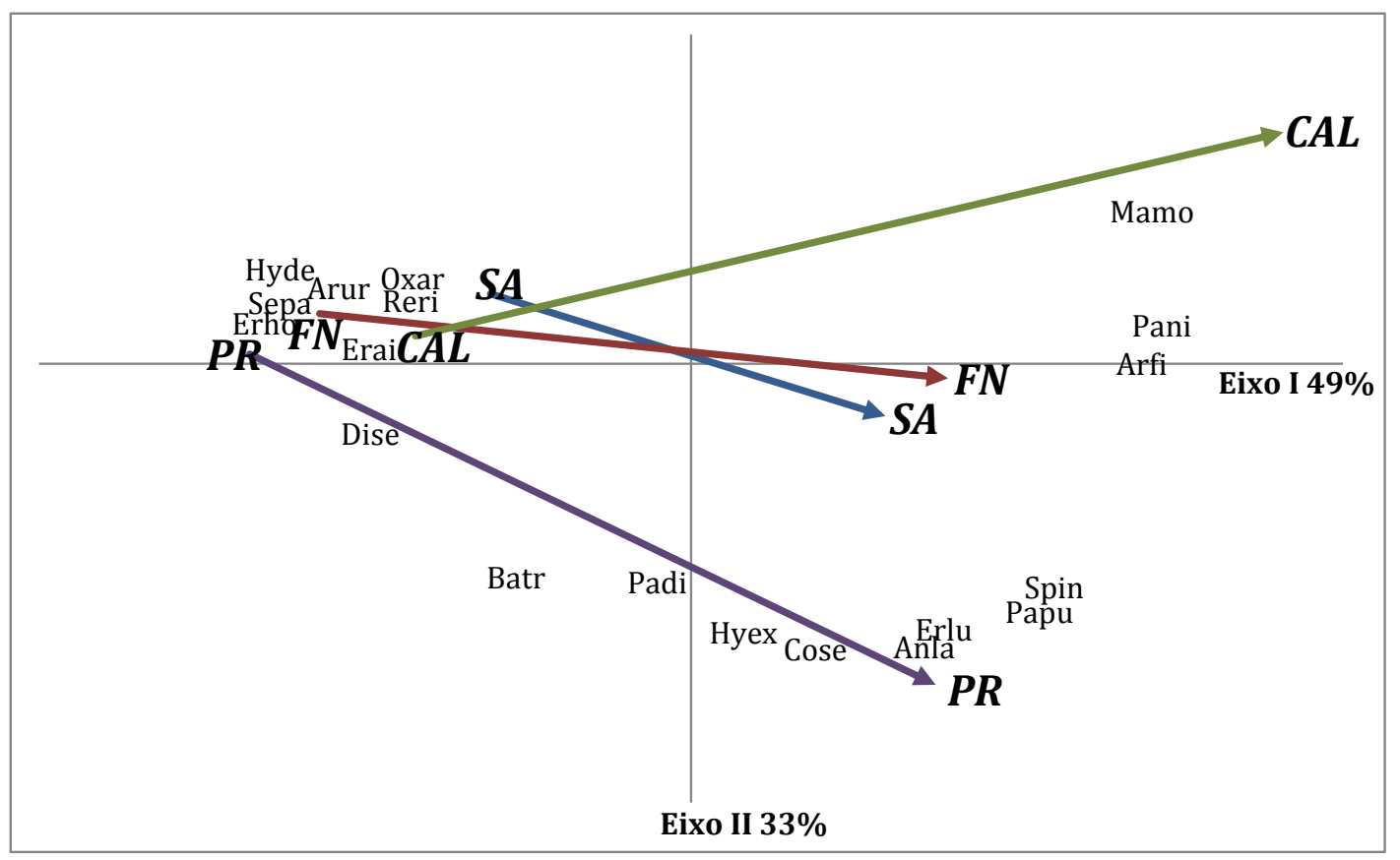

Figura 1. Diagrama de ordenação representando as trajetórias dos tratamentos no início e no final dos levantamentos, em função das espécies. Onde: $\mathrm{CAL}=$ calcário, $\mathrm{FN}=$ Fosfato natural, $\mathrm{SA}=$ sem adubação, $\mathrm{PR}=$ pó-de-rocha, Mamo= material morto, $\mathrm{Pani}=$ Paspalum nicorae, Arfi= Aristida filifolia, $\mathrm{Spin}=$ Sporobulus indicus, $\mathrm{Papu}=$ Paspalum pumilum, Erlu= Eragrostis lugens, Anla $=$ Andropogon lateralis, Cose $=$ Coelorachis selloana, Hyex= Hydrocotile exigua, Padi= Paspalum dilatatum, Batr $=$ Baccharis trimera, Dise $=$ Dichondra sericea, Erai $=$ Eragrostis airoides, Sepa $=$ Setaria parviflora, Arur $=$ Aristida uruguayensis, Oxar $=$ Oxalis articulata, $\mathrm{Hyde}=$ Hypoxis decumbens, Reri= Relbunium richardianum, Erho= Erygium horridum

cie Aristida laevis, que tem por característica não ser frequentemente pastejada, logo se associando com espécies mais consumidas, porém impossibilitando o acesso dos animais as mesmas, permitiu um acúmulo de forragem, que posteriormente não sendo utilizada, renasceu junto às touceiras.

Esta espécie foi representativa principalmente neste tratamento, embora a baixa correlação com os eixos 1 e 2, não permitiu sua representação no diagrama.

Os tratamentos fosfato natural e não adubado, teve trajetórias muito semelhantes e mais curtas, caracterizadas principalmente pelo aumento das espécies Aristida filifolia e Paspalum nicore, ambas descritas com hábito prostrado, o que é um aspecto diferenciado principalmente no que diz respeito a primeira espécie, que normalmente apresenta-se formando touceiras. Esse fato pode ser ainda consequência do histórico de manejo anterior das áreas.

Outro tratamento, caracterizado pela adubação com pó-de-rocha é representado principalmente pela diminuição da contribuição de algumas espécies como Sporobulus indicus, Paspalum dilatatum, Andropogon lateralis, Eragrostis lugens, todas caracterizadas por terem apresentado hábito cespitoso.

Na figura 2 estão representadas as espécies em função da adubação e do manejo do pastoreio (controlado e contínuo). O eixo de ordenação 1 explica $50 \%$ e o eixo $242 \%$, o que representa $92 \%$ da variação total.

Pode-se observar que os tratamentos, adubados com fosfato natural e os que não receberam adubação, que permaneceram em pastejo controlado, tem uma composição botânica bastante semelhante, tendo como principal representante das gramíneas a espécie Paspalum notatum, o que também foi observado por Castilhos (2002) que descreveu para áreas com ou sem adubo submetidas a pastejo esta espécie como sendo a de maior contribuição.

O tratamento sob pastejo contínuo e sem adubação esteve associadas com espécies estoloníferas e prostradas, dadas que se assemelham com os descritos por Trindade (2008), que demonstraram diferenças estruturais na vegetação dependentes de diferentes situações de pastejo, sendo áreas pastejadas continuamente caracterizadas por uma vegetação mais baixa.

Os tratamentos com pó-de-rocha (PRCNT) e calcário (CACNT) tiveram diferenças em suas composições florísticas, o tratamento com calcário e pastejo controlado (CACNT), tem como principal característica a alta associação com material morto, Aspilia montevidense, Senecio selloi e R. Richardianum.

No tratamento com pó-de-rocha pode ser observada uma maior associação com espécies de gramíneas, Paspalum pumilum, $P$. dilatatum, A. lateralis e Botriochloa 


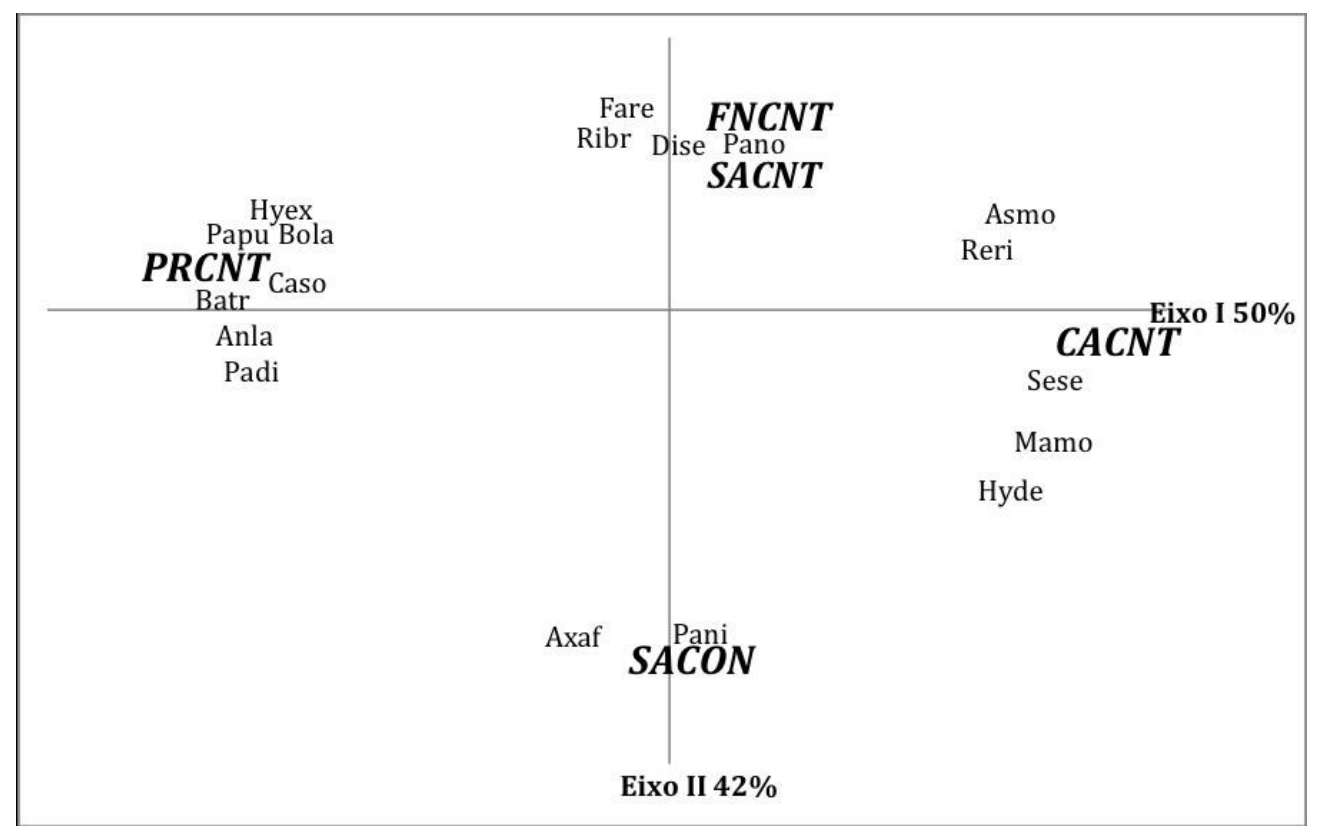

Figura 2. Diagrama de ordenação representando os tratamentos em função da adubação e manejo (pastejo). Onde: $\mathrm{CACNT}=$ calcário + pastejo controlado, $\mathrm{FNCNt}=$ Fosfato natural + pastejo controlado, $\mathrm{SACNT}=$ sem adubação + pastejo controlado, PRCNT= pó-de-rocha + pastejo controlado, SACON= sem adubação + pastejo contínuo. Onde Mamo= material morto, Pale $=$ Paspalum nicore, Padi $=$ Paspalum dilatatum, Sese $=$ Senecio selloi, $\mathrm{Papu}=$ Paspalum pumilum, Bola $=$ Botriochloa laguroides, $\mathrm{Anl}=\mathrm{a}=$ Andropogon lateralis, $\mathrm{Hyex}=\mathrm{Hydrocotile} \mathrm{exígua,}$ $\mathrm{Axaf}=$ Axonopus affinis, Batr $=$ Baccharis trimera, $\mathrm{Dise}=$ Dichondra sericea, $\mathrm{Asmo}=$ Aspilia montevidense, $\mathrm{Pano}=$ Paspalum notatum, Fare $=$ Facelis retusa, Oxar $=$ Oxalis articulata, Hyde $=$ Hypoxis decumbens, Reri $=$ Relbunium richardianum, Ribr= Richardia brasiliensis

laguroides sendo que das quatro, três foram descritas com hábito entouceirado, somente a primeira deve hábito mais prostrado, neste tratamento também pode ser observado a participação de espécies tipicamente descritas em ambientes úmidos (Boldrini, 2009), como Hydrocotile exigua e Carex sororia, isto pode ser explicado em parte porque algumas das unidades de avaliação da composição florística deste tratamento foram alocadas em áreas com maior retenção de umidade. Castilhos (2002) descrevendo a dinâmica vegetacional em áreas com ou sem adubações, caracterizou áreas submetidas à adubação com NPK e sob pastejo, com uma contribuição maior de $C$. sororia.

O tratamento que não recebeu adubação e não teve o pastejo controlado, esteve mais associado com espécies mais prostradas, como Axonopus affinis e P. nicore, caracterizadas por serem bem adaptadas ao pastoreio, confirmando outros estudos semelhantes em ambientes sob pastoreio (Trindade, 2008 e Castilhos, 2002).

Na figura 3 está representado um dendrograma de classificação com o objetivo de agrupar os dados em função da adubação e pastejo (fig. 2). Onde fica evidente a distinção de quatro grupos nítidos obtidos pelo teste de nitidez dos grupos, sendo o primeiro representado pelos tratamentos que receberam adubação com fosfato natural e os que não receberam adubação, ambos com pastoreio controlado, um segundo grupo composto pelo

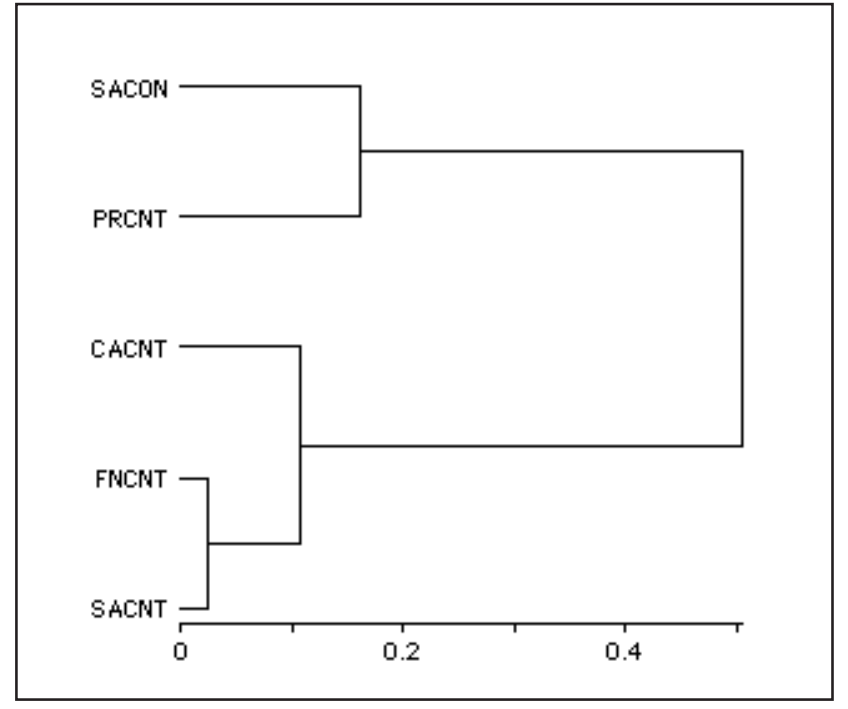

Figura 3. Dendrograma de classificação dos tratamentos conforme a composição e dinâmica de espécies em função dos tratamentos, obtido por análise de agrupamento pelo método da soma de quadrados. Onde SACON= sem adubação e pastejo sem controle; PRCNT= adubação com pó-de-rocha e pastejo controlado; $\mathrm{CACNT}$ = adubação com calcário e pastejo controlado; FNCNT = adubação com fosfato natural e pastejo controlado e SACNT= sem adubação e pastejo controlado. 

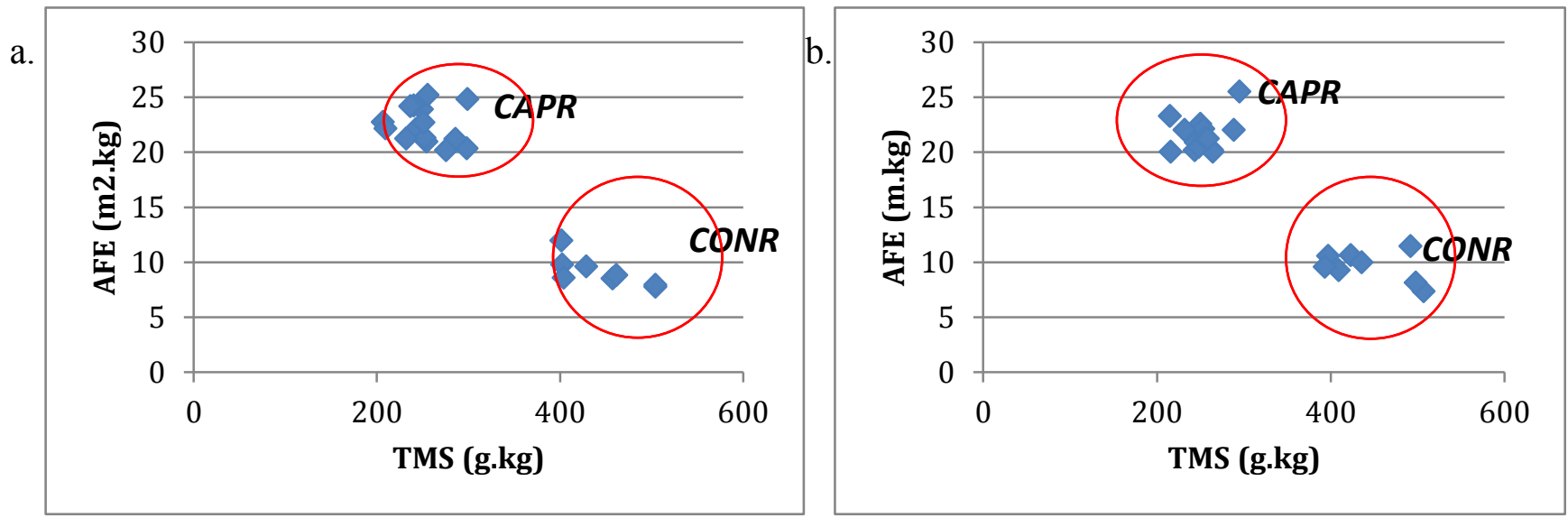

Figura 4. Gráficos representando as espécies (pontos) em função do Teor de matéria seca no primeiro levantamento (a) e no segundo (b). Onde: $\mathrm{CAPR}=$ caracteriza espécies com estratégias de Captura de Recursos e CONR= Conservação de Recursos.

tratamento sob calcário e pastoreio controlado, outro sob pó-de-rocha e pastoreio controlado e um último grupo com o tratamento que não recebeu adubação e permaneceu sem o controle do pastoreio.

Na figura 4, os gráficos a e b apresentam os valores de área foliar específica (AFE) e teor de matéria seca (TMS) na primeira coleta e última coleta de atributos. Podemos observar que os valores permanecem consistentes e com poucas variações nos dois períodos avaliados. Observa-se que as houve um agrupamento de espécies pelos altos valores de AFE como, por exemplo, P. notatum, A. affinis e Dichantelium sabulorum e outras pelos valores de TMS, como A. laevis, , Piptochaetium montevidense.

Nesta figura também podemos observar a separação de espécies por suas estratégias ecológicas de captura e conservação de recursos, as primeiras caracterizam-se por possuírem alto valor de área foliar específica e baixo teor de matéria seca representadas principalmente pelos grupos A e B descritos por Cruz et al., 2010, já as plantas descritas como conservadoras de recursos possuem um relação inversa, com baixa área foliar específica e alto teor de matéria seca, estas incluem-se nos tipos C e D

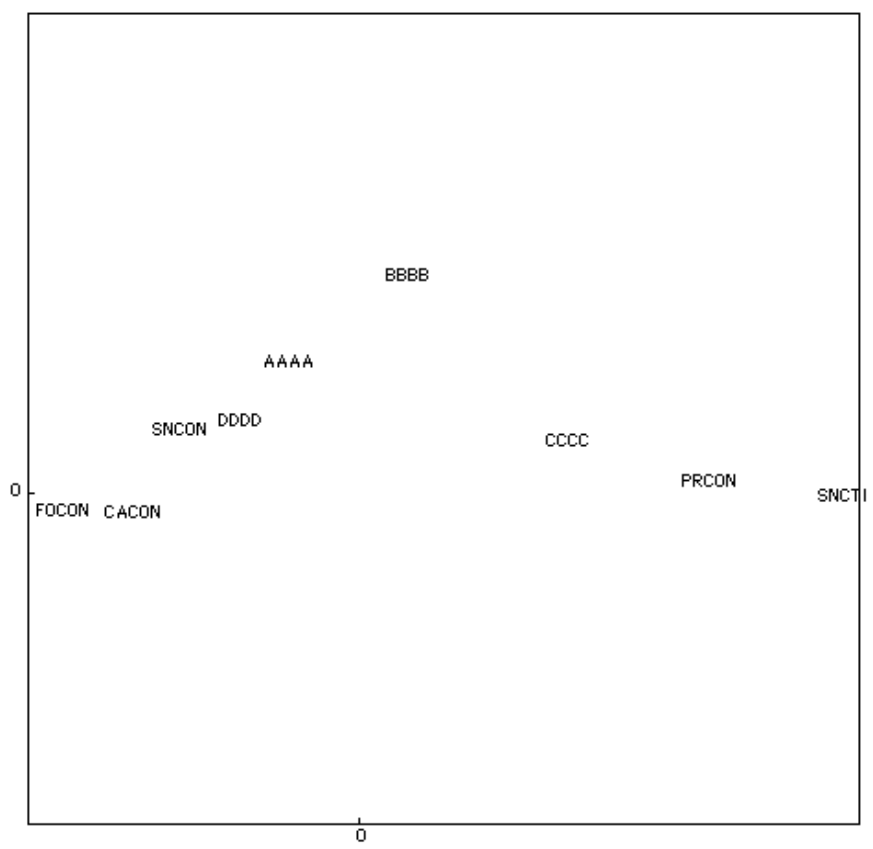

Figura 5. Tipos funcionais A (AAAA), B (BBBB), C (CCCC), D (DDDD), descritos nos diferentes tratamentos FOCON= fosfato e pastoreio controlado, $\mathrm{PRCON=}$ pó-de-rocha e pastoreio controlado, $\mathrm{CACON=}$ calcário e pastoreio controlado, $\mathrm{SNCON}$ = sem adubação e pastoreio controlado, $\mathrm{SNCTI}=$ sem adubação com pastoreio contínuo. 
(Cruz et al., 2010).

Com o objetivo de um maior entendimento da vegetação foram coletadas espécies da família Poaceae para determinação de área foliar específica e teor de matéria seca, estes valores foram utilizados para realizar o agrupamento das espécies em tipos funcionais, porém como os valores encontrados mantiveram-se dentro dos intervalos descritos por Cruz et al., 2010, e como não houve congruência entre os atributos coletados e as variáveis analisadas no presente trabalho, optou-se pela substituição dos valores determinados neste estudo pelos tipos funcionais (A, B, C e D), determinados por Quadros et al., 2009.

Através de uma análise de ordenação com esta substituição foi originada a Figura 4, onde não foi evidenciada associação nítida entre os tipos funcionais e os diferentes tratamentos, demonstrando que não houve uma diferenciação dos valores dos atributos entre os tratamentos e a ocorrência dos tipos funcionais foi semelhante nos tratamentos, fincando evidente a necessidade de mais coletas de atributos e a incluir a medição de outros atributos das espécies, visando uma associação mais nítida entre o ambiente e os tipos funcionais. Também se evidencia que a região tem ampla diversidade de tipos, apesar do histórico de manejo e falta de adubação.

Segundo o agrupamento de espécies em tipos funcionais representados na figura 5 , as espécies $A$. affinis, $D$. sabulorum, $P$. notatum e $P$. pumilum estiveram no grupo A, caracterizado por plantas bem adaptadas ao pastejo, que apresentam vantagem competitiva em ambiente fértil e que permaneça sob desfolhações frequentes, maioria espécies prostradas com alta AFE baixas TMS.

No grupo B estão representadas as espécies B. laguroides, C. selloana, S. microstachyum, adaptadas a pastejos mais controlados (moderados) e a maiores intervalos de desfolhações, apresentam um valor menor de AFE e maior TMS que as espécies pertencentes ao grupo A.

As espécies pertencentes ao $C$ são A. lateralis, $P$. montevidense e $S$. indicus, respresentando um grupo com menor adaptação ao pastejo, principalmente a pastejos intensos e frequentes, ou seja, as espécies são pouco adaptadas a desfolhações em menores intervalos de tempo apresentam valores maiores de TMS e menores de AFE do que as espécies pertencentes ao grupo B.

Finalmente as espécies pertencentes ao grupo D, sendo representado principalmente por espécies cespitosas, com altos valores de TMS e baixos de AFE, neste grupo no presente trabalho foram descritas as espécies $A$. laevis e novamente $P$. montevidense, sendo a última pertencente a dois grupos o que pode ser explicado pela plasticidade fenotípica que as espécies apresentam ou por estarem em diferentes estágios de desenvolvimento (Liancourt, 2005 e Soares et. al., 2011).

\section{Conclusões}

Ao longo dos períodos de avaliação foram observadas mudanças na dinâmica vegetacional em função da adubação e manejos do pastoreio, a partir de comunidades inicialmente similares.

Os valores de TMS e AFE mantiveram-se constantes nos dois períodos de avaliação e coletas, revelando a consistência dos atributos e permitindo agrupar as espécies em tipos funcionais já descritos.

Não houve uma associação nítida entre os tipos funcionais e os variáveis ambientes, necessitando de mais estudos, coletas e agregação de um maior número de atributos das espécies.

\section{Referências}

ANSQUER P., THEAU J.P., CRUZ P., VIEGAS J., AL HAJ Khaled R., DURU M. Caractérisation de la diversité fonctionnelle des prairies à flore complexe : vers la construction d'outils de gestion, Fourrages 2004. 179, p. 353-368.

BOLDRINI, I.I. A Flora dos Campos do Rio Grande do Sul. In: Pillar, V.P.; Müller, S.C.: Castilhos, Z.M.S.; Jacques, A.V. (Org.). Campos Sulinos - Conservação e Uso Sustentável da Biodiversidade. 2 ed. Brasilia, DF: MMA, 2009. v. 1, p. 63-77.

CARVALHO, P. C. F.; FISHER, V.; SANTOS, D. T.; RIBEIRO, A. M. L.; QUADROS, F. L. F.; CASTILHOS, Z. M. S.; POLI, C. H. E. C.; MONTEIRO, A. L. G.; NABINGER, C.; GENRO, T. C. M.; JACQUES, A. V. A. Produção animal no bioma Campos Sulino. Revista Brasileira de Zootecnia, v. 35, Supl. Esp., p. 156-202, 2006.

CASTILHOS, Z.M. S de. Dinâmica vegetacional e tipos funcionais em áreas excluídas e pastejadas sob diferentes condições iniciais de fertilização. 2002. 114p. Tese (Doutorado em Zootecnia) Universidade Federal do Rio Grande do Sul, Porto Alegre.

CORNELISSEN J. H. C., LAVOREL S., GARNIER E., DIAZ S., BUCHMANN N., GURVICH D. E., REICH P. B., ter STEEGE H., MORGAN H. D., van der HEIJDEN M. G. A., PAUSAS J. G. \& POORTER H.: A handbook of protocols for standardised and easy measurement of plant functional traits worldwide. - Austral. J. Bot. 51: 335-380. 2003

CRUZ, P. ; QUADROS, F. L. F. ; THEAU, J. P. ; FRIZZO, A. ; JOUANY, C. ; DURU, M.; CARVALHO, P. C. F. Leaf Traits as Functional Descriptors of the Intensity of Continuous Grazing 
in Native Grasslands in the South of Brazil. Rangeland Ecology \& Management, v. 63, p. 350358, 2010.

FLORES, J. e JURADO, E. Are nurse-protégé interactions more common among plants from arid environments? Journal of Vegetation Science, 14: 911-16. 2003.

GARNIER, E.; LAURENT, G.; BELLMANN, A.; DEBAIN, S.; BERTHELIER, P.; DUCOUT, B.; ROUMET, C.; NAVAS, M-L. Consistency of species ranking based on functional leaf traits. New Phytologist, v.152, p.69-83, 2001.

FERREIRA, E. T.; NABINGER, C.; ELEJALDE, D. A. G.; FREITAS, A. K. de; CARASSAI, I. J. ; SCHMITT, F. Fertilization and oversowing on natural grassland: effects on pasture characteristics and yearling steers performance. Revista Brasileira de Zootecnia. v. 40, p. 2039-2047, 2011.

LAVOREL, S.; GARNIER, E. Predicting changes in community composition and ecosystem functioning from plant traits: revisiting the Holy Grail. Functional Ecology, v.16, n.5, p.545- 556, 2002.

LIANCOURT, P.; CORCKE, E.; MICHALET, E. Stress tolerance abilities and competitive responses in a watering and fertilization field experiment. Journal of Vegetation Science 16: 713-722, 2005.

MARTINS, C. E. N. e QUADROS, F. L. F.. BOTANAL: desenvolvimento de uma planilha eletrônica para avaliação de disponibilidade de matéria seca e composição florística de pastagens. 2004. Anais In: REUNIÓN DEL GRUPO TÉCNICO REGIONAL DEL CONO SUR EN Mejoramiento y utilización de los recursos forrajeros del área tropical y subtropical - Grupo Campos, 2004, Salto. MEMORIAS. V. 1. p. 229-231.

MARTINS, C. E. N.; QUADROS, F. L. F.; GARAGORRY, F. C.; ROSSI, G. E.; TRINDADE, J. P. P. Implementação do componente espacial na planilha eletrônica BOTANAL. 2007. In: IV CONGRESSO NACIONAL SOBRE MANEJO DE PASTIZALES NATURALES E I CONGRESO DEL MERCOSUL SOBRE MANEJO DE PASTIZALES NATURALES, Vila Mercedes. Anais. Congresso Nacional Sobre Manejo de Pastizales Naturales. 2007. p. 1-1, v. 1.

PELLEGRINI, L. G. de; NABINGER, C.; NEUMANN, M.; CARVALHO, P. C. de F.; CRÂNCIO, L.

A. Produção de forragem e dinâmica de uma pastagem natural submetida a diferentes métodos de controle de espécies indesejáveis e à adubação. Revista Brasileira de Zootecnia. v. 39, p. 2380-2388, 2010.

PILLAR, V.D. Multivariate exploratory analysis and randomization testing with MULTIV. Coenoses 12: 145-148. 2009

PINTO, M. F. ; NABINGER, C.; BOLDRINI, I. I. ; FERREIRA, P.M.A. ; SETUBAL, R. B. ; TREVISAN, R. ; FEDRIGO, J. K. ; CARASSAI, I. J. . Floristic and vegetation structure of a grassland plant community on shallow basalt in Southern Brazil. Acta Botanica Brasílica. v. 27, p. 162-179, 2013

SCHÖNBACH, F.; WAN, H.; GIERUS, M.; BAI, Y.; MÜLLER, K.; LIN, L.; SUSENBETH, A.; TAUBE, F. Grassland responses to grazing: effects of grazing intensity and management system in an Inner Mongolian steppe ecosystem. Plant and Soil, v.340, p.103-115, 2010.

SOARES, A. B. ; CARVALHO, P. C. de F. ; NABINGER, C.; TRINDADE, J. P. P. TRINDADE, J. K. da ; MEZZALIRA, J. C. Dinâmica da composição botânica numa pastagem natural sob efeito de diferentes ofertas de forragem. Ciência Rural. v. 41, p. 1459-1465, 2011

RASBAND, W.S. 2007. ImajeJ., version 1.42q. National Institute of Health, USA.

TOTHILL, J.C., HARGREAVES, J.N.G., JONES, R.M., McDONALD, C.K. BOTANAL - A comprehensive sampling and computing procedure for estimating pasture yield and composition. 1. Field sampling. Tropical Agronomy Technical Memorandum 78: 24 p. 1992.

TRINDADE, J.P.P., QUADROS, F.L.F.; PILLAR, V.D.P. Vegetação campestre de areais do Sudoeste do Rio Grande do Sul sob pastejo e com exclusão de pastejo. Pesquisa Agropecuaria Brasileira. 43: 771-77, 2008 